OPEN ACCESS

Edited by:

Yu Xiao,

Hunan Agricultural University, China

Reviewed by:

Fei Xia,

Shaanxi University of Science and

Technology, China

Dingfu Xiao,

Independent Researcher,

Changsha, China

Liang Zhao,

Washington State University,

United States

Xi Ma,

China Agricultural University, China

*Correspondence:

Yizhen Wang

yizhenwang321@zju.edu.cn

Specialty section:

This article was submitted to

Food Chemistry,

a section of the journal

Frontiers in Nutrition

Received: 08 December 2021

Accepted: 21 January 2022

Published: 28 February 2022

Citation:

Wang C, Wei S, Jin M, Liu B, Yue M and Wang $Y$ (2022) Integrated Microbiomic and Metabolomic

Dynamics of Fermented Corn and

Soybean By-Product Mixed

Substrate. Front. Nutr. 9:831243.

doi: 10.3389/fnut.2022.831243

\section{Integrated Microbiomic and Metabolomic Dynamics of Fermented Corn and Soybean By-Product Mixed Substrate}

\author{
Cheng Wang 1,2,3,4,5, Siyu Wei ${ }^{1,2,3,4,5}$, Mingliang Jin ${ }^{1,2,3,4,5}$, Bojing Liu ${ }^{1,2,3,4,5}$, Min Yue ${ }^{6}$ and \\ Yizhen Wang ${ }^{1,2,3,4,5 *}$
}

${ }^{1}$ National Engineering Laboratory for Feed Safety and Pollution Prevention and Controlling, Hangzhou, China, ${ }^{2}$ Key Laboratory of Molecular Animal Nutrition, Ministry of Education, Hangzhou, China, ${ }^{3}$ Key Laboratory of Animal Nutrition and Feed Science (Eastern of China), Ministry of Agriculture and Rural Affairs, Hangzhou, China, ${ }^{4}$ Key Laboratory of Animal Feed and Nutrition of Zhejiang Province, Hangzhou, China, ${ }^{5}$ Institute of Feed Science, Zhejiang University, Hangzhou, China, ${ }^{6}$ Institute of Preventive Veterinary Sciences and Department of Veterinary Medicine, Zhejiang University College of Animal Sciences, Hangzhou, China

Microbes and their metabolites produced in fermented food have been considered as critical contributors to the quality of the final products, but the comprehensive understanding of the microbiomic and metabolomic dynamics in plant-based food during solid-state fermentation remains unclear. Here, the probiotics of Bacillus subtilis and Enterococcus faecalis were inoculated into corn and defatted soybean to achieve the two-stage solid-state fermentation. A $16 \mathrm{~S}$ sequencing and liquid chromatography-tandem mass spectrometry were applied to investigate the dynamics of microbiota, metabolites, and their integrated correlations during fermentation. The results showed that the predominant bacteria changed from Streptophyta and Rickettsiales at O h to Bacillus and Pseudomonas in aerobic stage and then to Bacillus, Enterococcus, and Pseudomonas in anaerobic stage. In total, 229 notably different metabolites were identified at different fermentation times, and protein degradation, amino acid synthesis, and carbohydrate metabolism were the main metabolic pathways during the fermentation. Notably, phenylalanine metabolism was the most important metabolic pathway in the fermentation process. Further analysis of the correlations among the microbiota, metabolites, and physicochemical characteristics indicated that Bacillus spp. was significantly correlated with amino acids and carbohydrate metabolism in aerobic stage, and Enterococcus spp. was remarkably associated with amino acids metabolism and lactic acid production in the anaerobic stage. The present study provides new insights into the dynamic changes in the metabolism underlying the metabolic and microbial profiles at different fermentation stages, and are expected to be useful for future studies on the quality of fermented plant-based food.

Keywords: fermentation, metabolomic analyses, dynamics, microbiomic data, plant-based food by-product 


\section{INTRODUCTION}

Corn is one of the main grains produced worldwide, providing $30 \%$ of food calories for more than 4.5 billion people worldwide, and is considered to be the main staple food in most countries $(1,2)$. Whereas, compared with other cereal crops, the nutritional value of corn is low, lacking essential amino acids and lysine (3). Soy-derived foods have been consumed for centuries, especially in Asian diets. But soy products contain isoflavones, antigen proteins, and fiber substances that are not easy to be absorbed, which affect their bioavailability $(4,5)$. Therefore, many processing technologies like physical and chemical methods have been applied to corn and soybean-based foods to enhance the nutritional value of the final products. However, these processing tools are un-environmental friendly and lose nutrients (6).

Fermentation is one of the traditional biotechnologies in the food field because it makes a solid foundation for the development of safe food with better nutrition and functions. It is a natural way to preserve food, increase nutritional value and digestibility, and reduce anti-nutritional factors. Fermenting microbes like Bacillus and Aspergillus can degrade anti-nutritional factors and improve food quality $(7,8)$. Lactic acid bacteria can produce organic acids, improve food flavor, and lengthen storage time $(9,10)$. In the past few years, many studies have been conducted on fermentation to obtain fermented corn-based products that are beneficial to human health (11-13). In addition, fermentation has been used to improve the bioavailability of protein, vitamins, minerals, and isoflavones in soybeans, as well as to change their flavor, improve stability, and even create novel foods (14-17). Natto, fermented bean curd, Miso, and soy sauce have a long history in the Asian diet. It has been reported that these traditional fermented soybean-based foods can improve bone health, reduce the risk of cancer, and prevent the progression of diabetes (18).

Fermentation is conducted through the action of various microorganisms under open or semi-open conditions, including starters and local microorganisms, and plays important roles in anti-nutritional factor degradation, nutrition improvement, and flavor production $(19,20)$. At present, high-throughput sequencing provides a reliable method for the comprehensive description of microbial community dynamics and expands our understanding of the microbial community structure of fermented corn and soybean products. The latest research showed that Lactococcus, Streptococcus, Enterobacter, Acinetobacter, and fungi such as Trichosporon and Aspergillus are common fermenting microbes in Sufu flora (21). In addition, microbial abundance is mainly related to nutritional characteristics. Besides, metabolomics methods such as gas chromatography (GC), mass spectrometry (MS), and high-performance liquid chromatography (HPLC) have been applied to determine the metabolite profiles of fermented foods $(22,23)$. However, the comprehensive dynamic changes of microbes and metabolites during plant-based food fermentation still lack understanding, especially in corn and defatted soybean. Here, corn and defatted soybean were carried out with a two-stage solid-state fermentation inoculating with Bacillus subtilis and Enterococcus faecalis.
A $16 \mathrm{~S}$ rRNA sequencing and liquid chromatography-mass spectrometry (LC/MS) were used to explore the microbial and metabolic dynamics during the fermentation. The evidence will expand our knowledge of improving the quality of fermented plant-based food from an integrated microbiomic and metabolomic perspective.

\section{MATERIALS AND METHODS}

\section{Experimental Design and Sampling}

Bacillus subtilis (NCBI Accession No. MH885533, CGMCC No:12825) and Enterococcus faecalis (NCBI Accession No. MN038173, CGMCC 1.15424) were cultured, respectively, in Luria broth and de Man, Rogosa, and Sharpe liquid medium at $37^{\circ} \mathrm{C}$, as described in detail by Wang et al. (24). About $200 \mathrm{~g}$ of the substrates contained $40 \%$ of defatted soybean, $40 \%$ of corn, and $20 \%$ of yellow wine lees were transferred into a $500 \mathrm{~mL}$ Erlenmeyer flask. Sterilized water was added to achieve a $40 \%$ moisture concentration. B. subtilis $\left(1 \times 10^{7} \mathrm{CFU} / \mathrm{g}\right)$ was inoculated in the mixed substrate at $37^{\circ} \mathrm{C}$ for $24 \mathrm{~h}$ in aerobic fermentation, and then E. faecalis $\left(2 \times 10^{7} \mathrm{CFU} / \mathrm{g}\right)$ was inoculated in the fermented substrate with an anaerobic fermentation at $37^{\circ} \mathrm{C}$ until $48 \mathrm{~h}$. Samples were collected at 0 , 12,24 , and $48 \mathrm{~h}$. The nutrition determination of the fermented matrix is shown in Supplementary Table S1.

\section{The Measurement of Nutritional Content}

Moist samples $(\sim 100 \mathrm{~g})$ at $0,12,24$, and $48 \mathrm{~h}$ were collected to determine the numbers of microorganisms and microbial metabolites and for 16S rRNA gene sequencing, and the remaining samples were dried at $60^{\circ} \mathrm{C}$ for $24 \mathrm{~h}$, cooled, ground, and subjected to physicochemical analysis. Dried samples were collected for further analysis of the crude protein (CP), neutral detergent fiber (NDF), acid detergent fiber (ADF), and amylose contents using AOAC International guidelines (25). The content of phytate phosphorus was analyzed according to the method described by Shi et al. (26). The $\mathrm{pH}$ of the fermented product was determined with a $\mathrm{pH}$ meter (Mettler Toledo, Switzerland) according to a previous study (27). Lactate was detected using a lactic acid assay kit (Nanjing Jiancheng Bioengineering Institute, Nanjing) following the manufacturer's instructions. The contents of antigenic protein were analyzed using a commercial kit (Jiangsu Meibiao Biological Technology Co., Jiangsu, China).

\section{Liquid Chromatography-Mass Spectrometry Analysis and Data Processing}

A $50 \mathrm{mg}$ of the sample was weighted to an EP tube, and 1,000 $\mu \mathrm{L}$ extract solution (methanol:acetonitrile:water $=2: 2: 1$, with isotopically labeled internal standard mixture) was added. Then the samples were homogenized at $35 \mathrm{~Hz}$ for $4 \mathrm{~min}$ and sonicated for $5 \mathrm{~min}$ in ice water bath. The homogenization and sonication cycle was repeated 3 times. Then the samples were incubated for $1 \mathrm{~h}$ at $-40^{\circ} \mathrm{C}$ and centrifuged at $12,000 \mathrm{rpm}$ for $15 \mathrm{~min}$ at $4^{\circ} \mathrm{C}$. The resulting supernatant was transferred to a fresh glass vial for analysis. The quality control (QC) sample was prepared 
by mixing an equal aliquot of the supernatants from all of the samples.

LC-MS/MS analyses were performed using a UHPLC system (Vanquish, Thermo Fisher Scientific) with a UPLC BEH Amide column $(2.1 \mathrm{~mm} \times 100 \mathrm{~mm}, 1.7 \mu \mathrm{m})$ coupled to Q Exactive HFX mass spectrometer (Orbitrap MS, Thermo). The mobile phase consisted of $25 \mathrm{mmol} / \mathrm{L}$ ammonium acetate and 25 ammonia hydroxide in water $(\mathrm{pH}=9.75)(\mathrm{A})$ and acetonitrile $(\mathrm{B})$. The auto-sampler temperature was $4^{\circ} \mathrm{C}$, and the injection volume was $3 \mu \mathrm{L}$. The QE HFX mass spectrometer was used for its ability to acquire MS/MS spectra on information-dependent acquisition (IDA) mode in the control of the acquisition software (Xcalibur, Thermo). In this mode, the acquisition software continuously evaluates the full scan MS spectrum. The ESI source conditions were set as follows: sheath gas flow rate as $30 \mathrm{Arb}$, Aux gas flow rate as $25 \mathrm{Arb}$, capillary temperature $350^{\circ} \mathrm{C}$, full $\mathrm{MS}$ resolution as $60,000, \mathrm{MS} / \mathrm{MS}$ resolution as 7,500, collision energy as 10/30/60 in NCE mode, spray Voltage as $3.6 \mathrm{kV}$ (positive) or $-3.2 \mathrm{kV}$ (negative), respectively.

The raw data were converted to the mzXML format using ProteoWizard and processed with an in-house program, which was developed using R and based on XCMS, for peak detection, extraction, alignment, and integration. Then an in-house MS2 database (BiotreeDB) was applied in metabolite annotation. The cutoff for annotation was set at 0.3 . The obtained data were conducted to principal component analysis (PCA), Partial Least Squares Discriminant Analysis (PLS-DA), and orthogonal partial least squares discriminant analysis (OPLS-DA). The goodnessof-fit parameter (R2) and the predictive ability parameter (Q2) were used to evaluate the quality of PLS-DA and OPLSDA models. Variable importance projection (VIP) $>1.0$ and $P<0.05$ were identified as the differential metabolites. To further interpret the biological significance of metabolites, metabolic pathway analyses were performed by an online analysis platform in the MetaboAnalyst 5.0 (https://www.metaboanalyst. $\mathrm{ca} /$ ). KEGG analysis has been conducted by enrichment analysis sections of MetaboAnalyst.

\section{Bioinformatics Analysis of Sequencing Data}

Total DNA was extracted from 16 samples using the E.Z.N.A. soil DNA kit (Omega Bio-Tek, Norcross, GA, United States). A NanoDrop 2,000 UV-vis spectrophotometer (Thermo Scientific, Wilmington, DE, United States) and 1\% agarose gel electrophoresis were used to analyze DNA content and quality.

The MiSeq platform (Personal Biotechnology Co., Ltd, Shanghai, China) was used to describe the bacterial community based on the gene segment from the the V3V4 gene regions of the bacterial $16 \mathrm{~S}$ rRNA gene primers 338F (5'-ACTCCTACGGGAGGCAGCAG-3') and 806R (5' GGACTACHVGGGTWTCTAAT-3'). PCR was conducted as follows: $3 \mathrm{~min}$ of denaturation at $95^{\circ} \mathrm{C} ; 27$ cycles of $30 \mathrm{~s}$ at $95^{\circ} \mathrm{C}, 30 \mathrm{~s}$ for annealing at $55^{\circ} \mathrm{C}$, and $45 \mathrm{~s}$ for elongation at $72^{\circ} \mathrm{C}$; and a final extension at $72^{\circ} \mathrm{C}$ for $10 \mathrm{~min}$. The AxyPrep DNA gel extraction kit (Axygen Biosciences, Union City, CA, United States) and QuantiFluor-ST instrument (Promega, United States) were used to further extract, purify, and quantify the PCR products. Subsequently, raw Illumina FASTQ files were demultiplexed, quality filtered, and analyzed using Quantitative Insights into Microbial Ecology (QIIME v1.9.1). Raw fastq files were quality filtered by Trichromatic and merged by FLASH. OTUs were clustered with a $97 \%$ similarity cutoff using UPARSE (version 7.1). The taxonomy of each $16 \mathrm{~S}$ rRNA gene sequence was analyzed using the RDP Classifier algorithm (http://rdp. cme.msu.edu/) against the Silvia 16S rRNA database using a confidence threshold of $70 \%$. The assembled MiSeq sequences were submitted to the NCBI's Sequence Read Archive (SRA BioProject no. PRJNA552228) for open access. Estimates of diversity values for these samples using the Chao1, Shannon, and Simpson indexes for diversity estimation were calculated by rarefaction analysis. Nonmetric Multidimensional Scaling (NMDS) and cluster analysis with the ANOSIM method were conducted using the Web server tool METAGENassist based on unweighted UniFrac distances.

The main differentially abundant genera were selected by the LEfSe method (https://huttenhower.sph.harvard.edu/galaxy/). To predict metabolic genes during the process, PICRUSt (https:// huttenhower.sph.harvard.edu/galaxy/) was applied to obtain a functional profile from the $16 \mathrm{~S}$ rRNA data. Before metagenome prediction, the OTUs of 16S rRNA sequences were analyzed using PICRUSt. PICRUSt and KEGG were used to obtain functions for the genes that were predicted to be present in the samples and to assign the genes into metabolic pathways.

Spearman's rank correlation coefficient was calculated with $\mathrm{R}$ version 3.6.3 to evaluate the relationship among physicochemical characteristics, microbiota, and metabolites.

\section{Statistical Analysis}

All the data were presented as means $\pm \operatorname{SEM}(N=4$ for chemical and microbial analysis, $N=6$ for metabolic analysis). The data were analyzed applying the SPSS software (version 26.0, SPSS Inc. Chicago, IL, United States). Statistical differences between different fermentation stages were determined by Student's $t$ tests and one-way ANOVA followed by Duncan's multiple-range test. The $P$-values of the metabolomics and microbiome data were corrected using Welch's test and the Benjamini-Hochberg false-discovery rate (FDR). $P$-values $<0.05$ indicate statistically significant differences. Bar plots were generated in GraphPad Prism 8 (San Diego, CA, United States).

\section{RESULTS}

\section{The Change of Physicochemical Parameters and Microbial Community}

The nutrients are presented in Supplementary Table S1. The pH value showed a dramatic decrease from aerobic to anaerobic fermentation stage while the content of lactic acid increased approximately three-fold range at $48 \mathrm{~h}$. The crude protein (CP) content significantly increased from 28.21 to $31.54 \%$, and a sharp increase was observed in small peptides (SP). Notably, similar significantly downward trends were observed for the levels of amylose, NDF, phytate phosphorus, glycinin, and $\beta$-conglycinin during the whole process of fermentation.

The composition of the bacterial community during corn and defatted soybean fermentation is shown in Figure 1. 

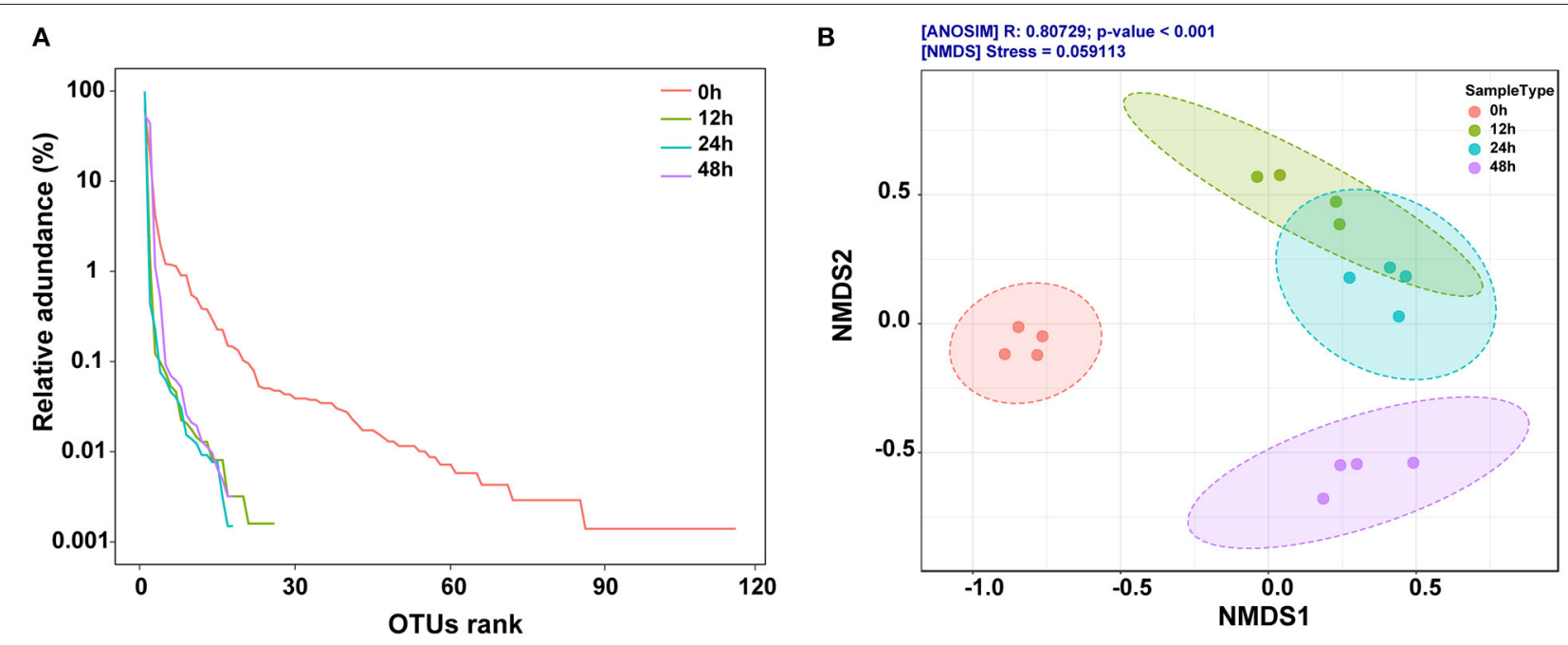

C

D
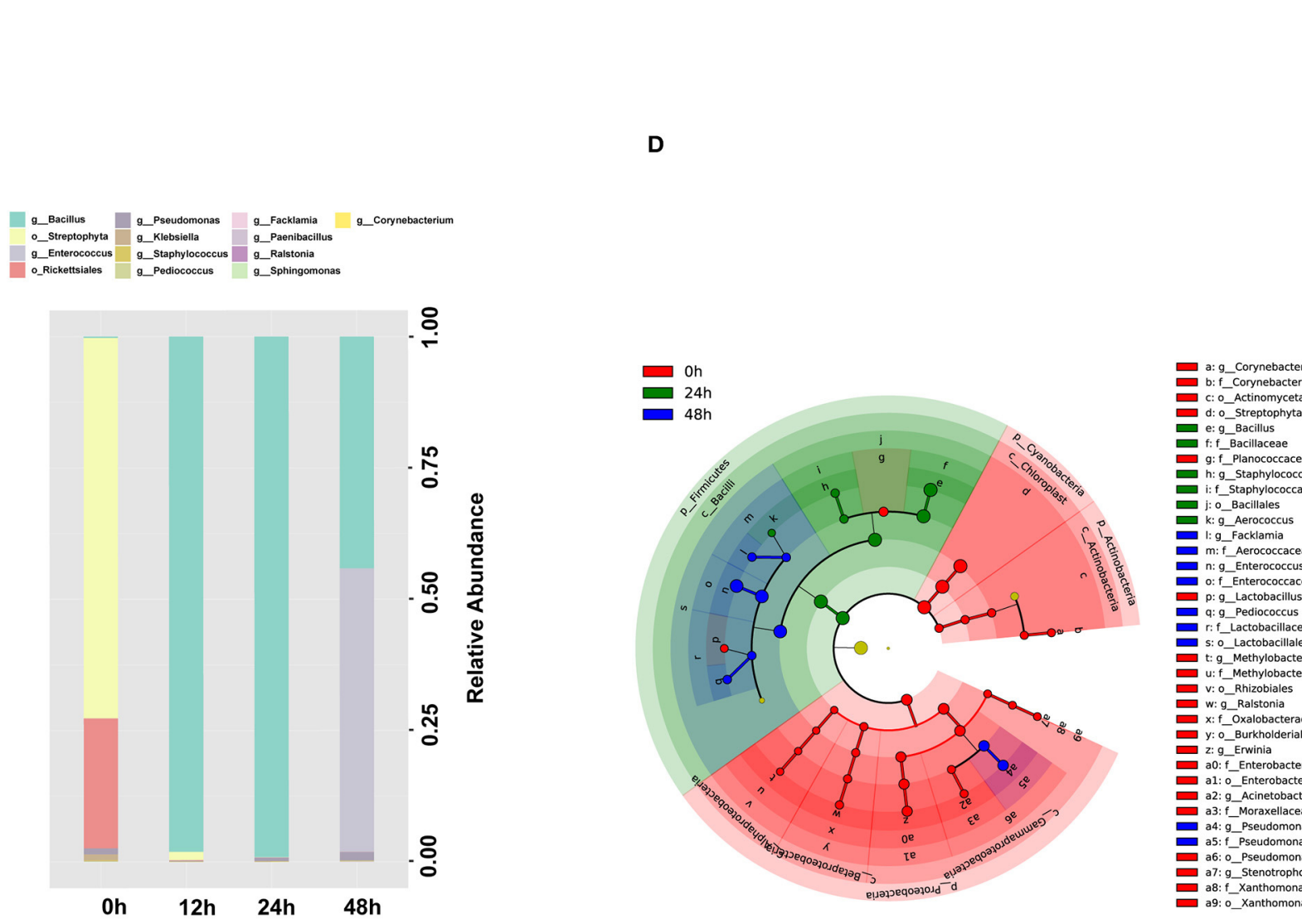
result showed that microbial $\beta$-diversity based on NMDS and Jaccard index distance method was distinct at different time points since the structures of microbial communities were separated into four clusters (Figure 1B). The results showed that the predominant bacteria changed from Streptophyta and Rickettsiales at $0 \mathrm{~h}$ to Bacillus and Pseudomonas in the aerobic stage and then to Bacillus, Enterococcus, and Pseudomonas in the anaerobic stage (Figure 1C). Significantly different bacteria from order to genus level among different fermentation time points were identified by the linear discriminant analysis effect size (LEfSe) (Figure 1D). The abundance of Bacillus, Staphylococcus, and Aerococcus increased significantly at $24 \mathrm{~h}$. Enterococcus, Pseudomonas, Pediococcus, and Facklamia were the predominant genus at $48 \mathrm{~h}$.

\section{Cluster Analysis of Metabolites at Different Fermentation Time Points}

Unsupervised mode of the PCA can reveal the variation between and within groups, and reflect the tendency of distribution as well as possible discrete points. The first principal component $(\mathrm{PC} 1=67.0 \%)$ and the second principal component $(\mathrm{PC} 2=16.5 \%)$ directly showed the similarities and differences between groups (Supplementary Figure S1A). The result showed that fermentation samples at $0,12,24$, and $48 \mathrm{~h}$ were separated, especially by the first principal component, and all samples fell within a 95\% confidence ellipse. The accurate differences between samples could not be completely interpreted according to the visual distinctions generated by the PCA plot, because PCA is an unsupervised model and belongs to exploratory analysis. Therefore, supervised classification models of Partial Least Squares Discriminant Analysis (PLS-DA) and Orthogonal Partial Least Squares Discriminant Analysis (OPLS-DA) were implemented to confirm the transformation degree of metabolites with fermentation time. The results showed obvious separation between different fermentation groups indicating that it could be used to identify the metabolic differences in the fermentation process of the substrates (Supplementary Figures S1B,C). A further cluster analysis was to determine whether metabolites differences existed during the fermentation. The chart showed that all the samples were clustered into four groups, and each cluster with samples belonged to the same time points (Supplementary Figure S1D). These results suggested that good stability and reproducibility were obtained, and the change of metabolites during fermentation was time dependent.

\section{The Changes of Metabolic Composition at Different Levels}

Metabolome analysis revealed that nucleosides, nucleotides, and analogs; lipids and lipid-like molecules; carbohydrates; amino acids, peptides, and proteins were the main abundant metabolites during the whole fermentation stage (Figure 2A). The relative abundance of amino acids, peptides, and proteins in the process of fermentation constantly increased $(P<$ 0.05), whereas the abundance of nucleosides, nucleotides, and analogs and lipids and lipid-like molecules maintained decreasing $(P<0.05)$. Carbohydrates decreased from 0 to $12 \mathrm{~h}$ and then remained in a relatively stable state. To find the specific change of the related metabolites, we explored the next level of top 20 metabolites (Figure 2B). At $0 \mathrm{~h}$, daidzin, lysoPC(16:0), glycerophosphocholine, lysoPC $[18: 2(9 \mathrm{Z}, 12 \mathrm{Z})]$, and 5-aminopentanoic acid were the main metabolites. The top 5 metabolites at $12 \mathrm{~h}$ were daidzin, norvaline, lysoPC $[18: 2(9 \mathrm{Z}, 12 \mathrm{Z})], \quad 2$-O-methyladenosine, and 5-aminopentanoic acid. Norvaline, 3,3,5-triiodo-Lthyronine-beta-D-glucuronoside, 5-aminopentanoic acid, imidazole-4-acetaldehyde, lysoPC[18:2(9Z,12Z)], and 2'-Omethyladenosine were the dominant substances at $24 \mathrm{~h}$. Notably, the concentration of metabolites was sharply elevated in the anaerobic stage. Phenylacetaldehyde dramatically raised in the anaerobic stage and became the predominant metabolites. 3,3,5-Triiodo-L-thyronine-beta-D-glucuronoside and norvaline in samples at $48 \mathrm{~h}$ were higher relative to that in $24 \mathrm{~h}$. During the whole fermentation stage, daidzin continued to reduce while 5 -aminopentanoic acid persistently increased.

\section{Different Metabolites in the Process of Fermentation}

The main metabolites based on the variable importance for the projection (VIP) were selected and sorted to better understand the changes of the primary different metabolites. Totally 229 metabolites were identified after annotation, and these metabolites have individual metabolic features at different time points after fermentation. Top 35 significantly different metabolites during fermentation were selected (Figure 3A), and the VIP values of these 35 metabolites based on the PLS-DA model were shown (Figure 3B). The main significantly different metabolites enriched in $0 \mathrm{~h}$ were trimethylamine $\mathrm{N}$-oxide, 1,3,5-trihydroxybenzene, cytidine $2^{\prime}, 3^{\prime}$-cyclic phosphate, isoferulic acid 3-sulfate, N'hydroxyneosaxitoxin, kojibiose, cyclic AMP, and apigenin 7-O-(6"-O-acetylglucoside). These eight metabolites slightly reduced from 0 to $12 \mathrm{~h}$ while remarkably decreased in 24 and $48 \mathrm{~h}(P<0.05)$. Fumigaclavine, deterrol stearate, and fumitremorgin B were the metabolic biomarkers at $24 \mathrm{~h}$. A total of 24 metabolites were significantly up-regulated at $48 \mathrm{~h}$, in particular, nnal-N-oxide, 4-(nitrosoamino)-1-(3-pyridinyl)1-butanone, and 9,10-epoxyoctadecanoic acid were the top 3 substrates, which had the high VIP scores. Phenylalanylasparagine, (E)-4-[5-(4-hydroxyphenoxy)-3-penten-1-ynyl] phenol, 9,10-epoxyoctadecanoic acid, cinnzeylanine [10]dehydrogingerdione, $(\mathrm{x})-2$-heptanol glucoside, and blumenol $\mathrm{C}$ glucoside were the most abundant compounds identified at $48 \mathrm{~h}$.

\section{Prediction of Metabolic Pathways by Identifying Significantly Different Metabolites}

KEGG databases were used to explore the metabolism pathway and elucidate the mechanism of metabolic changes during fermentation. Thus, enrichment analysis and topological analysis were performed to find the key pathways that were most relevant to the metabolites. A total of 22 metabolic pathways involving 229 different metabolites were sorted out throughout the fermentation process (Supplementary Tables S2, S3). 


\section{A}

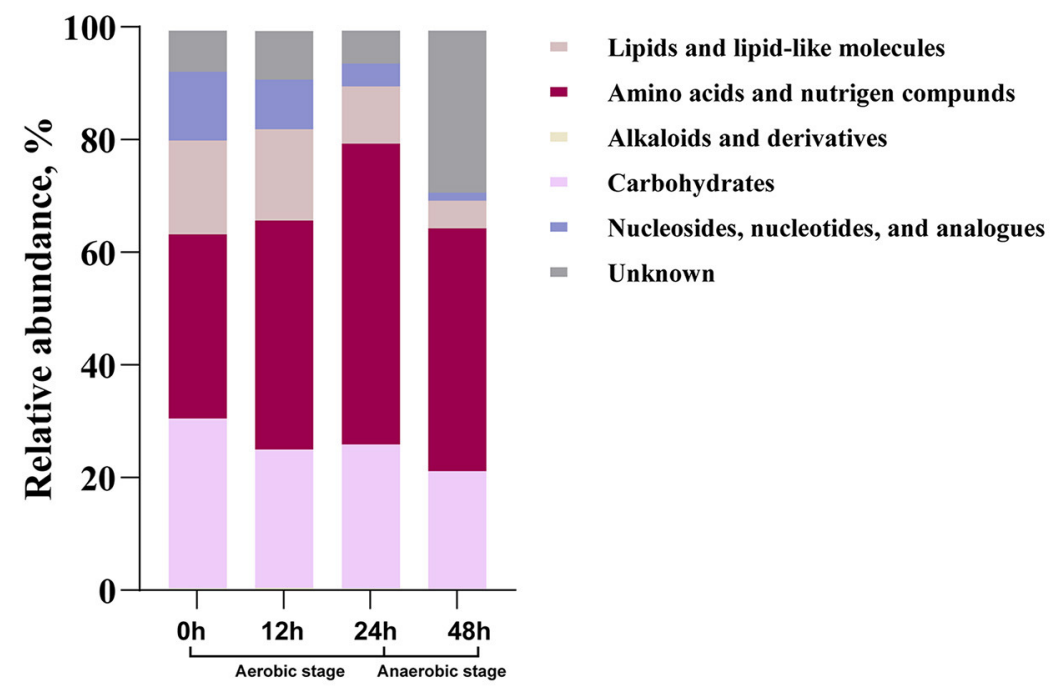

B

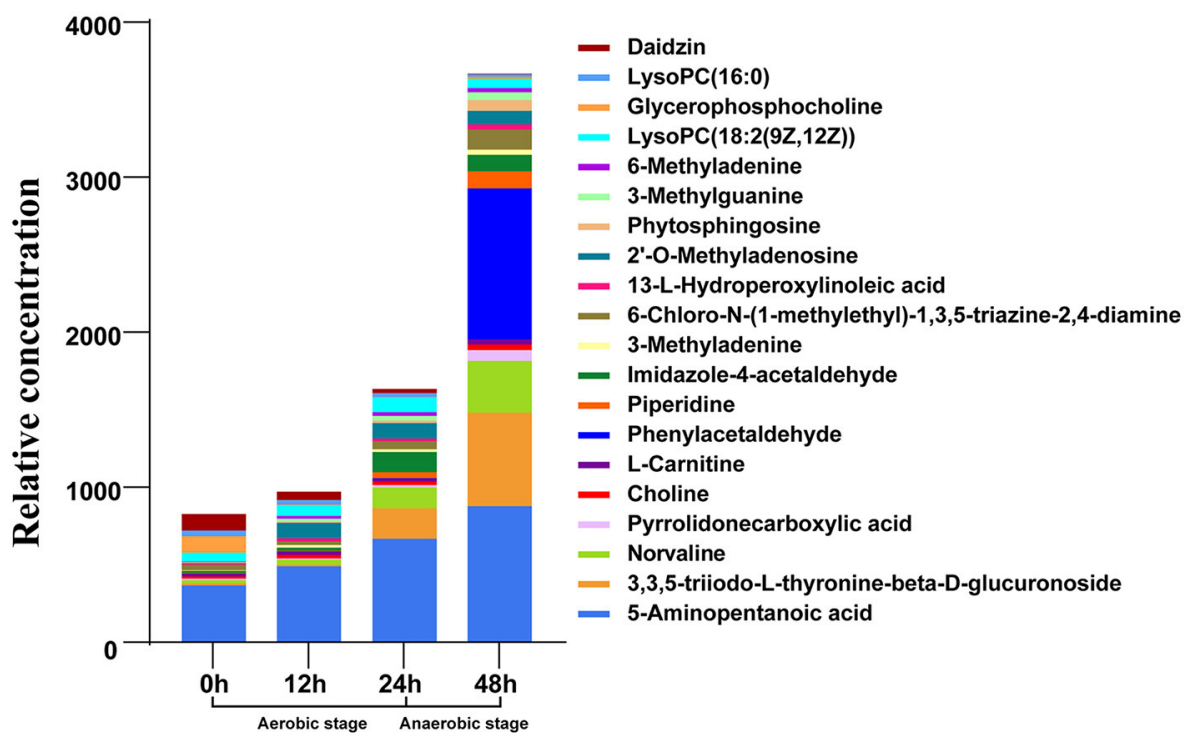

FIGURE 2 | The changes of metabolic composition at different levels in the process of fermentation. (A) Relative compositions of the main metabolites. (B) Top 20 metabolites at different fermentation times.

To more intuitively and directly compare the differences of metabolic pathways at different fermentation time points, the first 20 metabolic pathways were retained (Figure 4). Metabolites mapped to phenylalanine metabolism, starch and sucrose metabolism, taurine and hypotaurine metabolism, biotin metabolism, and cysteine and methionine metabolism were the main metabolism pathways in the whole fermentation process. Starch and sucrose metabolism and purine metabolism enriched in $0 \mathrm{~h}$ and decreased until the end of fermentation. Taurine and hypotaurine metabolism, glycerolipid metabolism, and glycerophospholipid metabolism were significantly upregulated in $24 \mathrm{~h}$. A total of 15 significantly different metabolic pathways were remarkably increased in $48 \mathrm{~h}$, including phenylalanine metabolism, biotin metabolism, cysteine and methionine metabolism, arginine biosynthesis, and glutathione metabolism etc. These findings suggested the succession of different 
A

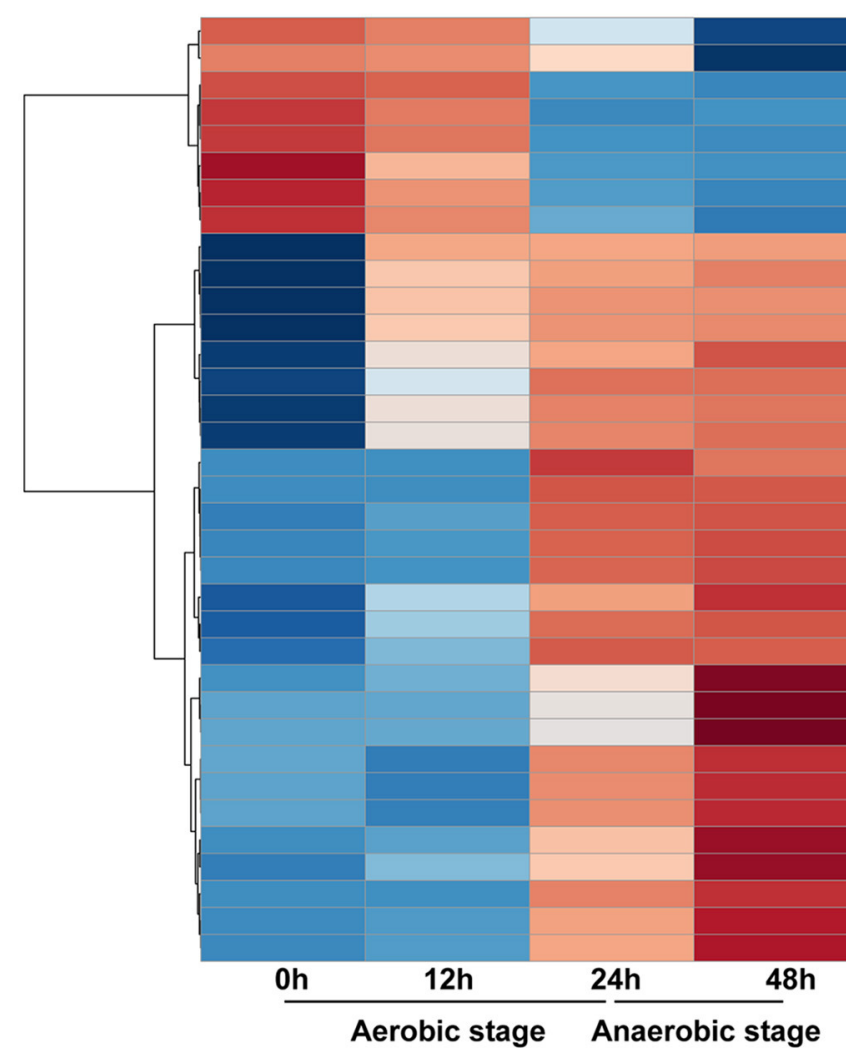

Trimethylamine $\mathrm{N}$-oxide

1,3,5-Trihydroxybenzene Cytidine 2', 3'-cyclic phosphate

Isoferulic acid 3-sulfate $\mathrm{N}$-Hydroxyneosaxitoxin Kojibiose Cyclic AMP

A pigenin 7-0-(6"-0-acetylglucoside)

(E)-Urocanic acid

3-Oxooctadecanoic acid Protein serine

[6]-Gingerdiol 3,5-diacetate

Nnal-N-oxide

Tsugaric acid C

Erinacine $\mathrm{C}$

17-alpha-Estradiol-3-glucuronide

Fumitremorgin $B$

Fumigaclavine $A$

Isoleucyl-Histidine

4-(Nitrosoamino)-1-(3-pyridinyl)-1-butanone

Tetraacetylethylenediamine

L-Kynurenine

Capryloylglycine

Deterrol stearate

Phenylalanyl-Asparagine

(E)-4-[5-(4-Hydroxyphenoxy)-3-penten-1-ynyl]phenol

9,10-epoxyoctadecanoic acid

Thiomorpholine 3-carboxylate

Cincassiol B

Hydroxysintaxanthi 5,6-epoxide

Cinnzeylanine

[10]-Dehydrogingerdione

Celastrol

(x)-2-Heptanol glucoside

Blumenol C glucoside

B

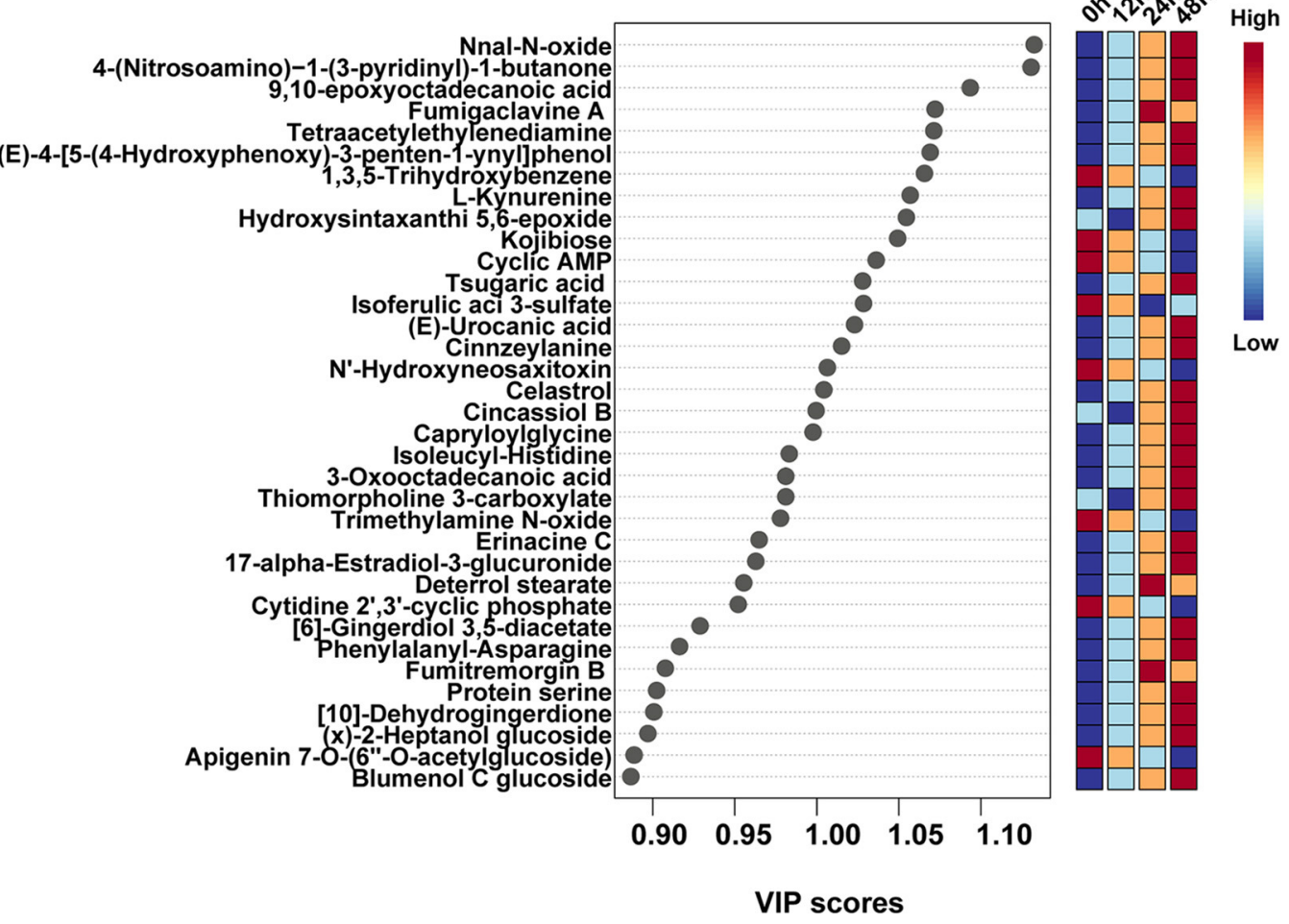

FIGURE 3 | Significantly different metabolites during fermentation. (A) Top 35 significantly different metabolites. (B) A scatter plot of the top 35 distinct metabolites was identified by applying variable importance projection (VIP). 


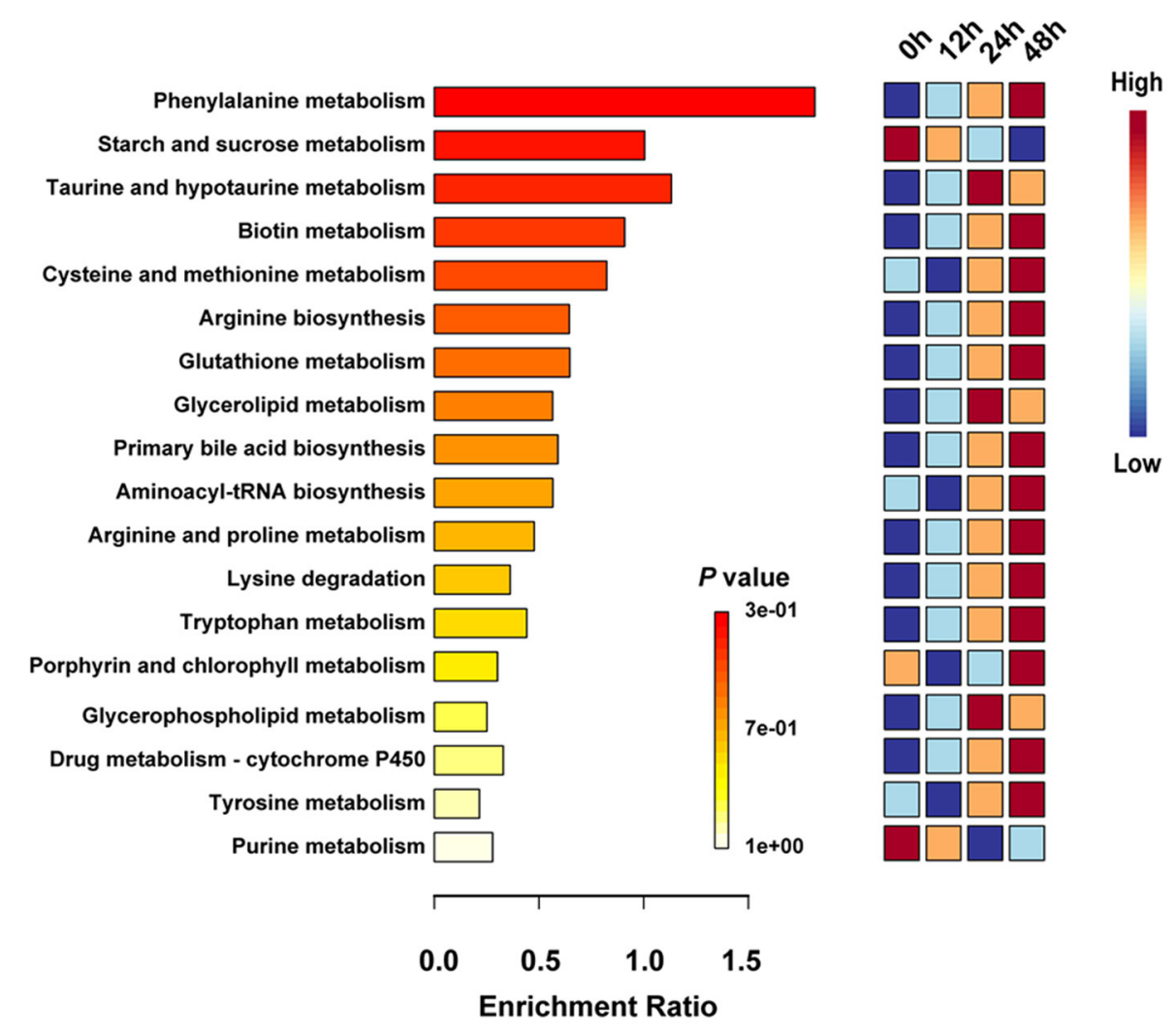

FIGURE 4 | Prediction of metabolic pathways by identifying significantly different metabolites. The significantly different metabolites are based on the variable importance for the projection (VIP) $>1$ and $P<0.05$.

metabolic pathways during the process of fermentation, especially the transformation of related metabolism caused by the change of fermentation condition.

\section{Correlations Among the Significantly Different Microbiota, Nutritional Value, Metabolites, and Metabolic Pathways During Fermentation}

To further investigate the observations regarding the impact of the changes in the bacteria, nutritional indexes, metabolites, and metabolic pathways, correlation analyses were performed. Bacillus, Staphylococcus, and Aerococcus which increased at $24 \mathrm{~h}$ had the opposite relations with nutritional value and metabolites, compared with that of $0 \mathrm{~h}$ enriched microbes. Bacillus, Staphylococcus, and Aerococcus were positively related with amino acids and nitrogen compounds and negatively correlated with lipids and nucleosides, and contributed to the nutritional value of the substrates (Figure 5A). Notably, Bacillus was negatively related to carbohydrates. Correlations among the metabolites, microbiota, and nutrients in the anaerobic stage are shown in Figure 5B. Enterococcus and Facklamia had positive correlations with crude protein, small peptides, and lactic acids, and were negatively correlated with most of the metabolites. The correlations between microbes and metabolic pathways were further conducted. Bacillus was negatively related to starch and sucrose metabolism and positively correlated with most of the amino acid metabolism in the aerobic stage (Figure 5C). In the anaerobic stage, Enterococcus was positively related to amino acid metabolism (Figure 5D).

\section{Integrated Microbiomic and Metabolomic Changes of Functional Pathways During Fermentation}

The integrated metabolic pathways are shown in the metabolome view map (Figure 6). A total of 6 microbial metabolism pathways based on KEGG database at level 1 were identified, including carbohydrate metabolism, lipid metabolism, amino acid metabolism, metabolism of cofactors and vitamins, metabolism of other amino acids, and glycan biosynthesis and metabolism. Carbohydrates metabolism consistently increased from the aerobic to anaerobic stages, while the metabolism of cofactors and vitamins, glycan biosynthesis, and metabolism decreased from 0 to $48 \mathrm{~h}$. Lipid metabolism and metabolism of other amino acids were enriched at $12 \mathrm{~h}$, amino acid metabolism increased to a high level at $24 \mathrm{~h}$. At level 2, starch and sucrose metabolism and glyoxylate and dicarboxylate metabolism showed an opposite expression trend of change. Primary bile acid biosynthesis and glycerolipid metabolism consistently increased, but glycerophospholipid metabolism decreased 


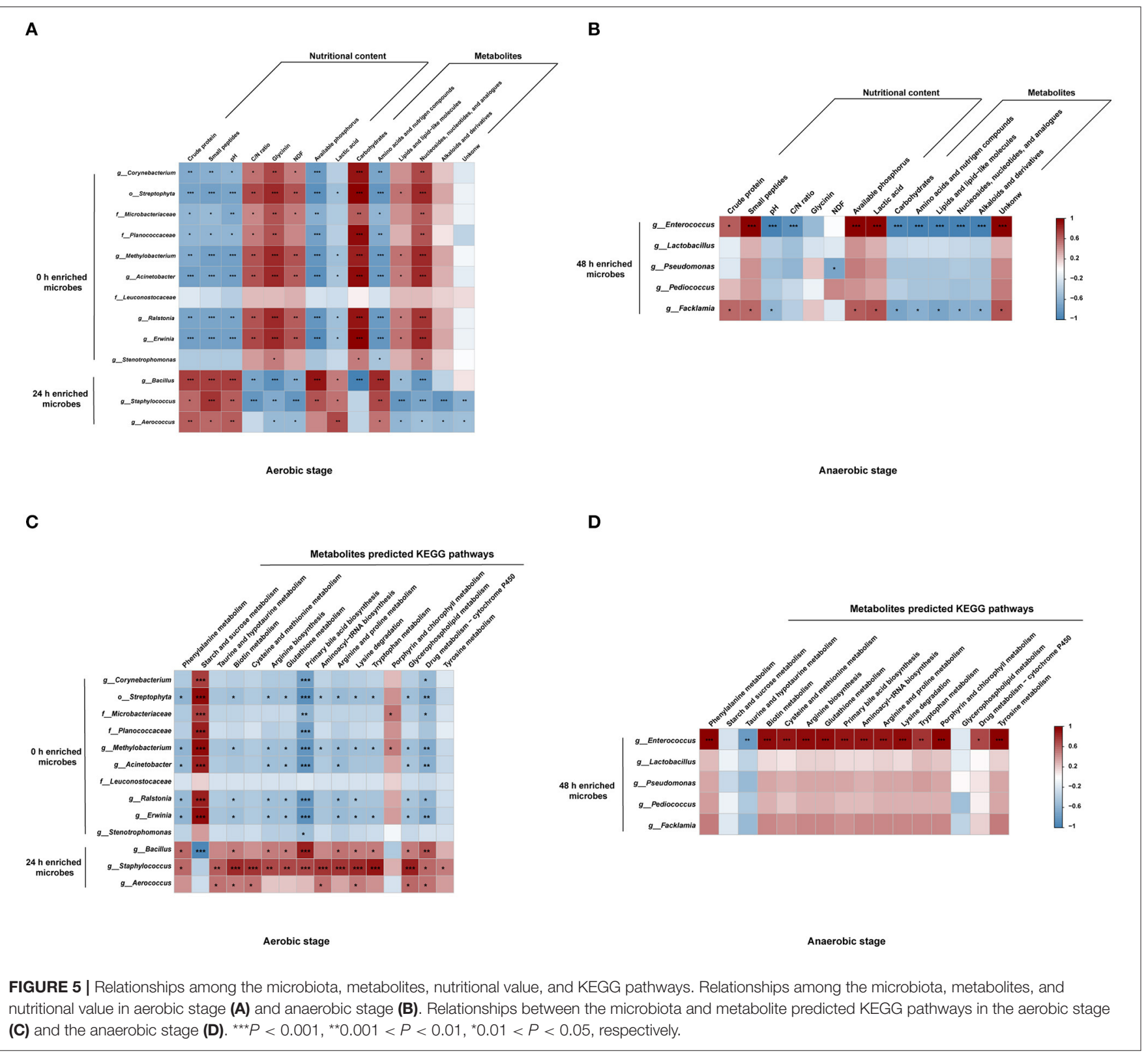

from 0 to $48 \mathrm{~h}$. C-lysine was the product of three major metabolic pathways involving carbohydrate metabolism, lipid metabolism, and amino acid metabolism, and then could be converted into 5-amino-levulinate in porphyrin and chlorophyll metabolism. Phosphatidyl-ethanolamine was produced by glycerophospholipid metabolism, and further generaterd ethanolamonephosphate which was higher at $12 \mathrm{~h}$. The content of L-cysteine and taurocholate was down-regulated, and Lcysteine was the precursor of taurine which can be converted into taurocholate.

\section{DISCUSSION}

In recent years, fermented plant-based foods have gained attention due to their potential health benefits $(28,29)$.
Fermented corn and soy-based foods such as fermented corn flour, Natto, Sufu, Miso, Douchi, and soy sauce are popular because they are important plant-derived food for humans and have a huge global consumption. Many research studies have reported that fermentation is capable of reducing antinutritional factors, making the original substrate more flavorful and nutritional. However, few studies spotlight the dynamics of fermented corn and soybean by-products by appling microbiome and matebolome. The present study indicated the temporal changes during microbial fermentation of fermented corn and defatted soybean based on using a multi-omics approach. Bacillus, Enterococcus, and Pseudomonas were mainly involved in the maturation of the fermented corn and defatted soybean. Importantly, phenylalanine metabolism was the main and vital metabolism pathway in the anaerobic fermentation stage. 


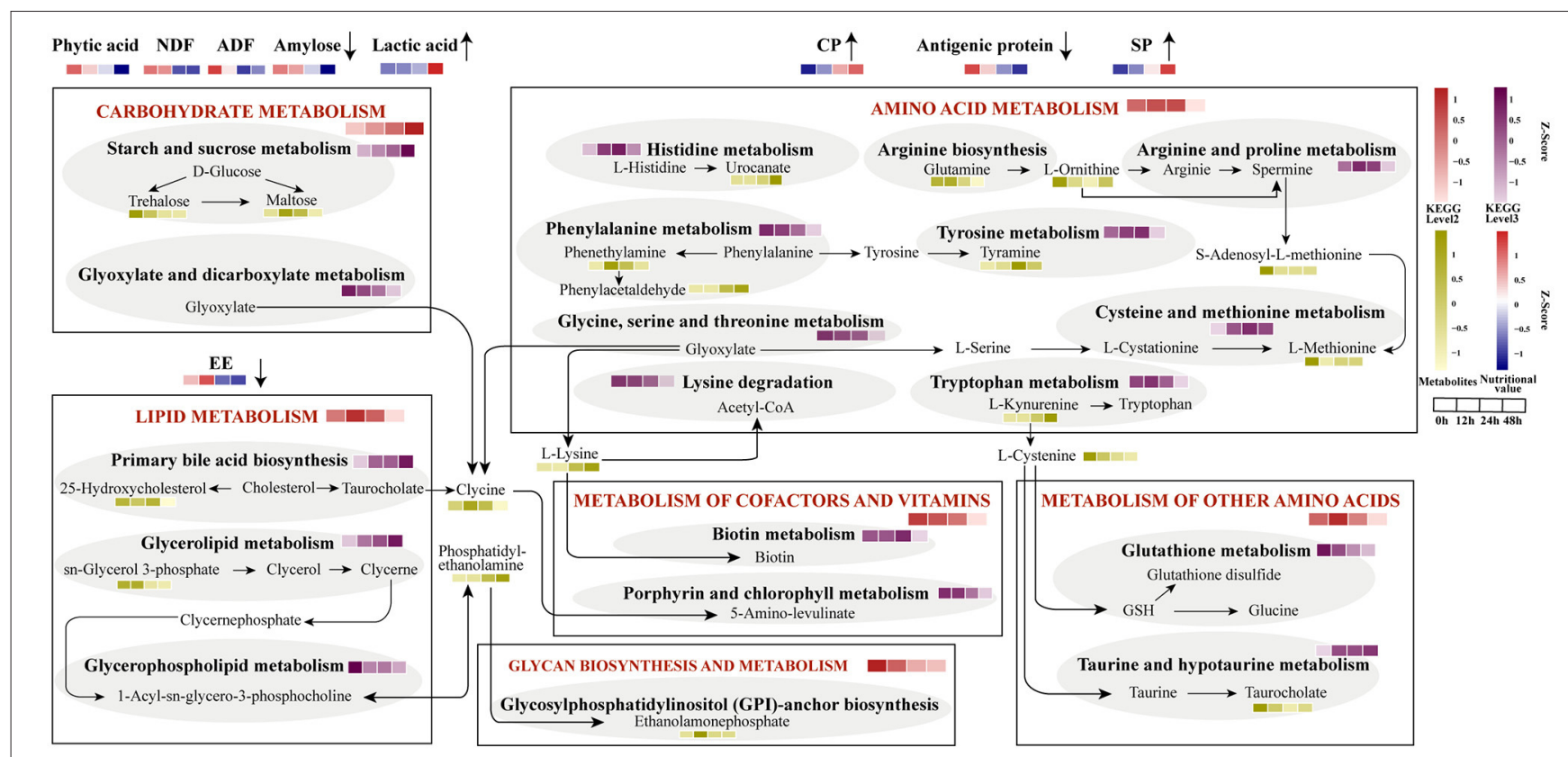

FIGURE 6 | Integrated microbiomic and metabolomic changes of functional pathways. The KEGG level2 was selected based on significantly different metabolic data. The abundance of KEGG level2 and 3 were predicted by $16 S$ data.

Correlation analysis suggested a strong relationship between Bacillus and amino acids in the aerobic stage, and Enterococcus mainly acted as an acid and amino acids producer at the anaerobic stage of fermentation.

The changes of metabolites were found to be time dependent during fermentation by adapting a two-step fermentation method. PLS-DA analysis and the structure of metabolites showed that metabolic profiles were significantly different among the groups. The microbial analysis also revealed that the dynamic changes of microbial community occurred during this two-stage solid-state fermentation process. These findings suggest that the change of metabolites during fermentation may be caused by the change of microbial niche at different stages. A recent study reported that different fermented soy foods had various soluble and volatile metabolites (30). Besides, fermenting microbes and metabolites contributes to the taste and flavor of Sufu (31). These findings have implications for considering the metabolites as a way to assess the fermented plant-based food and the metabolites can be used to understand the fermentation stages.

Metabolites annotation further indicated that metabolic activities of carbohydrates, amino acids, and lipid metabolism were dominant during fermentation, which is in agreement with previous studies about fermented soybean paste $(32,33)$. Amino acids, peptides, and proteins increased, whereas lipids and lipid-like molecules, and carbohydrates decreased during fermentation in our study. These results were consistent with the changes in nutrients as the content of antigenic protein, NDF, ADF, amylose, and crude fat reduced, and the level of crude protein raised during the anaerobic fermentation stage. For individual metabolites, daidzin, a natural organic compound in soybean (34), is elementary for the oxidation of acetaldehyde derived from ethanol metabolism and can be converted by resident bacteria for secondary metabolism (35). Soybean and soybean-based products are rich in daidzin, which is poorly absorbed in the human gut (36). The decrease of daidzin during the fermentation process in our study indicates that fermentation improves the bioavailability of isoflavones and assists in the digestion of protein. 5-Aminopentanoic acid is a lysine degradation product, which could be produced by bacterial catabolism of lysine (37). Nontargeted urine metabolomics analysis showed that 5-aminopentanoic acid was a biomarker, which played a role in protective and therapeutic effects on highfat feed-induced hyperlipidemia in rats (38). The increase of 5aminopentanoic acid suggests that the fermentation up-regulates potential benefit of the substrates. Notably, the dramatic increase in phenylacetaldehyde was observed at $48 \mathrm{~h}$. Phenylacetaldehyde is a typical fragrant compound in traditional Chinese-type soy sauce and alcohol-free beers $(39,40)$. These results provide an evidence that important contribution of these flavor compounds to the value of fermented corn and defatted soybean.

The identification of significantly different metabolites further confirmed that fermentation caused significant changes in various metabolites. Previous studies reported that several bacterial species (Bacillus and Pseudomonas) metabolically degraded trimethylamine oxide (TMAO) in food fermentation (41). Clinical evidence supported that there is a strong association between elevated TMAO levels with increased risk of developing diseases such as atherosclerosis and thrombosis (42). The present study demonstrated that the level of TMAO continued to decrease, and Bacillus and Pseudomonas were rich in the fermentation stage. These findings are consistent with previous reports and suggested that fermentation reduces 
harmful substances. The source of dipeptides and free amino acids is mainly caused by protease secreted by microorganisms that decompose the protein components of a Thai fermented soybean (43). Jin et al. (44) found that L-theanine, glutamine, and glutamic acid enriched in the fermented corn, defatted soybean, and bran incubated with a combination of probiotics. Studies showed that the concentrations of most amino compounds gradually increased during fermentation with soybean as substrate $(32,45)$. The concentration of phenylalanyl-asparagine, protein serine, and isoleucyl-histidine showed a steady increase over time, which supports the notion of a previous study. Phenylalanyl-asparagine and isoleucyl-histidine are incomplete breakdown products of protein digestion or protein catabolism. Phenylalanine, isoleucine, and histidine are essential aminoacids. Serine, a health-promoting compound, is functionally important in many proteins which are involved in the metabolism of fats, fatty acids, cell membranes, and a healthy immune system. These metabolites outcomes directly implied the degradation of protein during fermentation and the production of amino acids, peptides, and analogs occurred and may play essential roles in quality improvement of the fermented corn and defatted soybean.

To reveal the specific effect of the significantly different metabolites during fermentation, KEGG databases were used to characterize the most influential pathways. The level of phenylalanine and glutamine was improved during the fermentation of soybean $(46,47)$. Tyrosine is considered to be an indispensable dietary amino acid in humans and animals, and a diet supplemented with phenylalanine is a common way of compensating it (48). Glutathione is a major antioxidant, which is metabolized in multiple ways leading to the biosynthesis of glutamate, glycine, cysteine, and other amino acids (49). The present study showed that phenylalanine metabolism, glutathione metabolism, cysteine, and methionine metabolism were the top 3 important metabolism pathways during fermentation. Our previous study also demonstrated that the content of amino acid metabolism gradually increased as fermentation progressed in terms of the predicted microbial function (24). These results indicated that the metabolism of amino acids was active and the number of amino acids increased in the fermentation of corn and defatted soybean. In addition, phenylalanine metabolism is a major pathway for anthocyanin biosynthesis (50). Anthocyanin aroused a wide public concern as a potent beneficial metabolite due to its antioxidant activity, antimicrobial, antiviral, and antithrombotic characteristics $(51,52)$. Anthocyanin was detected after fermentation as well, suggesting that fermentation may improve the nutritional value of the fermented substances. Therefore, these affected metabolic pathways are closely related to the fermented corn and defatted soybean and provide clues for further study on the effect of two-stage fermentation on its metabolites.

Complex microbial-ecological interactions influence the production of metabolites. In the first-stage aerobic fermentation, Bacillus rapidly proliferated. Relevant studies reported that Bacillus was the predominant fermentative bacteria responsible for the natural fermentation of protein-rich food, production of flavor substances, conversion of complex food compounds in small components in the fermentation of west African seed condiments (53). In a Nigerian fermented soybean condiment, Bacillus was the dominant species occurring throughout the fermentation (54). Bacillus was positively related to amino acids and nitrogen compounds revealing that the proteolysis of Bacillus results in the increase of peptides, amino acids, and ammonia from proteins. In addition, the degradation of complex carbohydrates from the enzymes secreted by Bacillus, such as amylase, galactanase, galactosidase, glucosidase, and fructofuranosidase, interpreted the positive correlation between Bacillus and carbohydrates in the aerobic stage. Enterococcus, a lactate-producing bacteria as well as an acid-tolerant bacteria $(55,56)$, plays important roles in food production, particularly they can accelerate the ripening of food and improve the taste and flavor through proteolysis, lipolysis, carbohydrates breakdown, and the production of aromatic compounds (57). Enterococcus was the most dominant genus in the anaerobic fermentation stage. The enzyme secreted by Enterococcus and Bacillus played important roles in hydrolyzing proteins, and the accumulation of amino acids and related substances occurred in the whole process, resulting in a positive correlation between Enterococcus and amino acids. Small peptides in foods are generally easy to be absorbed and utilized by the gut, and most of them have specific biological functions (58-60), such as improving gut health and immunity. The relationships of microbes and metabolic pathways further confirmed the effects on protein and carbohydrates breakdown of Bacillus and amino acid synthesis of Enterococcus. A detailed understanding of the fermentation microbiota and their unique functional characteristics is fundamental to developing high-quality and safe fermented products and enhancing specifically adapted starter cultures. These results implied that Bacillus and Enterococcus were critical bacteria in corn and defatted soybean fermentation and suggested that they may be the optimized selective strains used in the two-stage fermentation.

The overview of the metabolic pathways map will help to further explain the causes of the differences in these metabolites and to explore the metabolic mechanism. Interestingly, the content of amino acid metabolism increased at the aerobic phase but declined abruptly at $48 \mathrm{~h}$, and the consistent increase of carbohydrates was observed according to the predicted microbial functions. These findings were distinct from the metabolomics analysis, suggesting the limitation and deviation of the analysis of the dynamics during the fermentation based solely on microbial data. In the first stage, bacteria like Bacillus which directly participated in amino acid metabolism resulted in increased metabolic capacity. With the proliferation of carbohydratedegrading bacteria and the increased acidification in the second stage, the main metabolism of fermentation gradually changes to carbohydrate metabolism. Therefore, the distinctive dominant metabolic functions at different fermentation stages were further interpreted. The physicochemical features were consistent with the change of metabolites, verified the metabolism differences caused by the significantly different bacteria in the two-stage fermentation. The metabolic process of fermented plant-based food is complex, and the specific metabolic mechanisms of these metabolites identified in our study are still not fully 
understood. Thus, future studies should focus on the secondary metabolites and establish standardized metabolic fingerprints for the fermentation.

\section{CONCLUSIONS}

In summary, the dynamic changes in the nutritional properties, microbial composition, and metabolites during the corn and defatted soybean fermentation were systematically studied. Phenylalanine metabolism, glutathione metabolism, and cysteine and methionine metabolism were considered to be the important metabolic pathways affecting the quality of fermented corn and defatted soybean. The results further unraveled that Bacillus spp. was the predominant genera that mainly participated in the breakdown of protein and carbohydrates in the aerobic stage, and Enterococcus spp. was associated with amino acid metabolism and lactic acid production toward the end of fermentation. This study potentially serves as a foundation for increasing the nutrition of corn and defatted soybean food and guides the underlying fermenting mechanism of solid-state fermentation of plant-based food.

\section{DATA AVAILABILITY STATEMENT}

The datasets presented in this study can be found in online repositories. The names of the repository/repositories and accession number(s) can be found in the article/Supplementary Material.

\section{AUTHOR CONTRIBUTIONS}

CW and YW conceived the project and designed the experiments. CW and SW analyzed the nutritional value, $16 \mathrm{~S}$ rRNA sequencing data and metabolic data, and wrote the manuscript. MJ, MY, and YW help review, revise, and approve the manuscript. BL assisted the nutritional value

\section{REFERENCES}

1. Nguyen H, Mwangomba W, Nyirenda EM. Delving into possible missing links for attainment of food security in Central Malawi: farmers' perceptions and long term dynamics in maize (Zea mays L) production. Heliyon. (2021) 7:e07130. doi: 10.1016/j.heliyon.2021.e07130

2. Jones RB, Berger PK, Plows JF, Alderete TL, Millstein J, Fogel J, et al. Lactose-reduced infant formula with added corn syrup solids is associated with a distinct gut microbiota in Hispanic infants. Gut Microbes. (2020) 12:1813534. doi: 10.1080/19490976.2020.1813534

3. Terefe ZK, Omwamba MN, Nduko JM. Effect of solid state fermentation on proximate composition, antinutritional factors and in vitro protein digestibility of maize flour. Food Sci Nutr. (2021) 9:6343-52. doi: 10.1002/fsn3.2599

4. Cao ZH, Green-Johnson JM, Buckley ND, Lin QY. Bioactivity of soy-based fermented foods: a review. Biotechnol Adv. (2019) 37:22338. doi: 10.1016/j.biotechadv.2018.12.001

5. Fernandez-Raudales D, Hoeflinger JL, Bringe NA, Cox SB, Dowd SE, Miller MJ, et al. Consumption of different soymilk formulations differentially affects the gut microbiomes of overweight and obese men. Gut Microbes. (2012) 3:490-500. doi: 10.4161/gmic. 21578 analyses and contributed to revise and approve the manuscript. All authors contributed to the article and approved the submitted version.

\section{FUNDING}

This work was funded by China Agriculture Research System of MOF and MARA (CARS-35).

\section{ACKNOWLEDGMENTS}

We are grateful to Shanghai Biotree Biotech Co., Ltd. and Yan Wang for their contributions to the LC-MS/MS analyses using their UHPLC system metabolite annotation and related bioinformatics analysis.

\section{SUPPLEMENTARY MATERIAL}

The Supplementary Material for this article can be found online at: https://www.frontiersin.org/articles/10.3389/fnut.2022. 831243/full\#supplementary-material

Supplementary Figure S1 | Dimension reduction analysis and clustering of dynamic metabolome. (A) Principal component analysis (PCA) plot of compounds in fermented mixed substances. (B) Partial Least Squares Discriminant Analysis (PLS-DA) plot of compounds in fermented mixed substances. The accuracy, goodness-of-fit (R2), and goodness-of-prediction (Q2) were 1.0, 0.999, and 0.991, respectively. (C) Orthogonal partial least-squares discrimination analysis (OPLS-DA) plot of compounds in fermented mixed substances; R2X, R2Y, and Q2 were $0.098,0.883$, and 0.523 , respectively. (D) Cluster analysis of different fermentation times.

Supplementary Table S1 | Determined nutrient contents of fermented corn and defatted soybean at $0,12,24$, and $48 \mathrm{~h}$.

Supplementary Table S2 | Identification of significantly different metabolites during fermentation.

Supplementary Table S3 | Enrichment pathways of significantly different metabolites in fermented mixed substances.

6. Adebo OA, Oyedeji AB, Adebiyi JA, Chinma CE, Oyeyinka SA, Olatunde $\mathrm{OO}$, et al. Kinetics of phenolic compounds modification during maize flour fermentation. Molecules. (2021) 26:6702 doi: 10.3390/molecules 26216702

7. Kimura K, Yokoyama S. Trends in the application of Bacillus in fermented foods. Curr Opin Biotechnol. (2019) 56:3642. doi: 10.1016/j.copbio.2018.09.001

8. Zhang D, Wang Y, Shen SN, Hou Y, Chen YY, Wang TT. The mycobiota of the human body: a spark can start a prairie fire. Gut Microbes. (2020) 11:655-79. doi: 10.1080/19490976.2020.1731287

9. Rathod NB, Phadke GG, Tabanelli G, Mane A, Ranveer RC, Pagarkar $A$, et al. Recent advances in bio-preservatives impacts of lactic acid bacteria and their metabolites on aquatic food products. Food Biosci. (2021) 44:101440. doi: 10.1016/j.fbio.2021.101440

10. Feng T, Wang J. Oxidative stress tolerance and antioxidant capacity of lactic acid bacteria as probiotic: a systematic review. Gut Microbes. (2020) 12:1801944. doi: 10.1080/19490976.2020.1801944

11. Cui L, Li DJ, Liu CQ. Effect of fermentation on the nutritive value of maize. Int J Food Sci Tech. (2012) 47:755-60. doi: 10.1111/j.1365-2621.2011.02904.x

12. Forsido SF, Hordofa AA, Ayelign A, Belachew T, Hensel O. Effects of fermentation and malt addition on the physicochemical properties 
of cereal based complementary foods in Ethiopia. Heliyon. (2020) 6:e04606. doi: 10.1016/j.heliyon.2020.e04606

13. Hiran $P$, Kerdchoechuen $O$, Laohakunjit N. Combined effects of fermentation and germination on nutritional compositions, functional properties and volatiles of maize seeds. J Cereal Sci. (2016) 10:2566. doi: 10.1016/j.jcs.2016.09.001

14. Chiang SS, Pan TM. Antiosteoporotic effects of Lactobacillus-fermented soy skim milk on bone mineral density and the microstructure of femoral bone in ovariectomized Mice. J Agric Food Chem. (2011) 59:773442. doi: $10.1021 / \mathrm{jf} 2013716$

15. Ikeda Y, Iki M, Morita A, Kajita E, Kagamimori S, Kagawa Y, et al. Intake of fermented soybeans, natto, is associated with reduced bone loss in postmenopausal women: Japanese Population-Based Osteoporosis (JPOS) Study. J Nutr. (2006) 136:1323-8. doi: 10.1093/jn/136.5.1323

16. Rekha CR, Vijayalakshmi G. Bioconversion of isoflavone glycosides to aglycones, mineral bioavailability and vitamin B complex in fermented soymilk by probiotic bacteria and yeast. J Appl Microbiol. (2010) 109:1198208. doi: 10.1111/j.1365-2672.2010.04745.x

17. Singh P, Kumar R, Sabapathy SN, Bawa AS. Functional and edible uses of soy protein products. Compr Rev Food Sci F. (2008) 7:1428. doi: 10.1111/j.1541-4337.2007.00025.x

18. Kwon DY, Daily JW, Kim HJ, Park S. Antidiabetic effects of fermented soybean products on type 2 diabetes. Nutr Res. (2010) 30:1-13. doi: 10.1016/j.nutres.2009.11.004

19. Oh NS, Joung JY, Lee JY, Song JG, Oh S, Kim Y, et al. Glycated milk protein fermented with Lactobacillus rhamnosus ameliorates the cognitive health of mice under mild-stress condition. Gut Microbes. (2020) 11:164361. doi: 10.1080/19490976.2020.1756690

20. Hwang JH, Wu SJ, Wu PL, Shih YY, Chan YC. Neuroprotective effect of tempeh against lipopolysaccharide-induced damage in BV-2 microglial cells. Nutr Neurosci. (2019) 22:840-9. doi: 10.1080/1028415X.2018.1456040

21. Wan HF, Wang LP, Zhao Y, Li L. Analysis of microorganisms in two kinds of Jiajiang sufu based on high-throughput sequencing. J. Chin. Inst. Food Sci.Technol. (2020) 20, 278-285. doi: 10.16429/j.1009-7848.2020.08.034

22. Beaumont M, Paës C, Mussard E, Knudsen C, Cauquil L, Aymard P, et al. Gut microbiota derived metabolites contribute to intestinal barrier maturation at the suckling-to-weaning transition. Gut Microbes. (2020) 11:1268-86. doi: 10.1080/19490976.2020.1747335

23. Moy YS, Chou CC. Changes in the contents of sugars and organic acids during the ripening and storage of Sufu, a traditional oriental fermented product of soybean cubes. J Agric Food Chem. (2010) 58:12790-3. doi: 10.1021/jf1033653

24. Wang C, Shi C, Su W, Jin M, Xu B, Hao L, et al. Dynamics of the physicochemical characteristics, microbiota, and metabolic functions of soybean meal and corn mixed substrates during two-stage solid-state fermentation. mSystems. (2020) 5:e0050119. doi: 10.1128/mSystems.00501-19

25. Feldsine P, Abeyta C, Andrews WH, Committee AIM. AOAC International methods committee guidelines for validation of qualitative and quantitative food microbiological official methods of analysis. J AOAC Int. (2002) 85:1187200. doi: 10.1093/jaoac/85.5.1187

26. Shi C, Zhang Y, Yin Y, Wang C, Lu Z, Wang F, et al. Amino acid and phosphorus digestibility of fermented corn-soybean meal mixed feed with Bacillus subtilis and Enterococcus faecium fed to pigs. J Anim Sci. (2017) 95:3996-4004. doi: 10.2527/jas.2017.1516

27. Yoshihara T, Oikawa Y, Kato T, Kessoku T, Kobayashi T, Kato S, et al. The protective effect of Bifidobacterium bifidum G9-1 against mucus degradation by Akkermansia muciniphila following small intestine injury caused by a proton pump inhibitor and aspirin. Gut Microbes. (2020) 11:1385404. doi: 10.1080/19490976.2020.1758290

28. Masia C, Geppel A, Jensen PE, Buldo P. Effect of Lactobacillus rhamnosus on physicochemical properties of fermented plant-based raw materials. Foods. (2021) 10:573. doi: 10.3390/foods10030573

29. Wuyts S, Van Beeck W, Allonsius CN, Van Den Broek MFL, Lebeer S. Applications of plant-based fermented foods and their microbes. Curr Opin Biotechnol. (2020) 61:45-52. doi: 10.1016/j.copbio.2019.09.023

30. Lee SH, Lee S, Lee SH, Kim HJ, Singh D, Lee CH. Integrated metabolomics and volatolomics for comparative evaluation of fermented soy products. Foods. (2021) 10:2516. doi: 10.3390/foods10112516
31. Yao D, Xu L, Wu MN, Wang XY, Wang K, Li ZJ, et al. Microbial community succession and metabolite changes during fermentation of BS Sufu, the fermented black soybean curd by Rhizopus microsporus, Rhizopus oryzae, and Actinomucor elegans. Front Microbiol. (2021) 12:665826. doi: $10.3389 /$ fmicb.2021.665826

32. An FY, Li M, Zhao Y, Zhang Y, Mu DL, Hu XY, et al. Metatranscriptome-based investigation of flavor-producing core microbiota in different fermentation stages of dajiang, a traditional fermented soybean paste of Northeast China. Food Chem. (2021) 343:128509. doi: 10.1016/j.foodchem.2020.128509

33. Wu JR, Tian T, Liu YM, Shi YX, Tao DB, Wu RN, et al. The dynamic changes of chemical components and microbiota during the natural fermentation process in Da-Jiang, a Chinese popular traditional fermented condiment. Food Res Int. (2018) 112:457-67. doi: 10.1016/j.foodres.2018.06.021

34. Chiang CM, Wang DS, Chang TS. Improving free radical scavenging activity of soy isoflavone glycosides daidzin and genistin by 3'Hydroxylation using recombinant Escherichia coli. Molecules. (2016) 21:1723. doi: $10.3390 /$ molecules 21121723

35. Rafii F. The role of colonic bacteria in the metabolism of the natural isoflavone daidzin to equol. Metabolites. (2015) 5:56-73. doi: 10.3390/metabo5010056

36. Mustafa SE, Mustafa S, Abas F, Manap MYABD, Ismail A, Amid M, et al. Optimization of culture conditions of soymilk for equol production by Bifidobacterium breve 15700 and Bifidobacterium longum BB536. Food Chem. (2019) 278:767-72. doi: 10.1016/j.foodchem.2018.11.107

37. Cheng J, Luo Q, Duan HC, Peng H, Zhang Y, Hu JP, et al. Efficient whole-cell catalysis for 5-aminovalerate production from L-lysine by using engineered Escherichia coli with ethanol pretreatment. Sci Rep. (2020) 10:990. doi: 10.1038/s41598-020-57752-x

38. Zeng W, Huang KE, Luo Y, Li DX, Chen W, Yu XQ, et al. Nontargeted urine metabolomics analysis of the protective and therapeutic effects of Citri Reticulatae Chachiensis Pericarpium on high-fat feed-induced hyperlipidemia in rats. Biomed Chromatogr. (2020) 34:e4795. doi: 10.1002/bmc.4795

39. Piornos JA, Balagiannis DP, Methven L, Koussissi E, Brouwer E, Parker JK. Elucidating the odor-active aroma compounds in alcohol-free beer and their contribution to the worty flavor. J Agr Food Chem. (2020) 68:1008896. doi: 10.1021/acs.jafc.0c03902

40. Zhao GZ, Ding LL, Hadiatullah H, Li S, Wang XW, Yao YP, et al. Characterization of the typical fragrant compounds in traditional Chinese-type soy sauce. Food Chem. (2020) 312:126054. doi: 10.1016/j.foodchem.2019.126054

41. Osimani A, Ferrocino I, Agnolucci M, Cocolin L, Giovannetti M, Cristani C, et al. Unveiling hakarl: a study of the microbiota of the traditional Icelandic fermented fish. Food Microbiol. (2019) 82:56072. doi: $10.1016 /$ j.fm.2019.03.027

42. Simo C, Garcia-Canas V. Dietary bioactive ingredients to modulate the gut microbiota-derived metabolite TMAO. New opportunities for functional food development. Food Funct. (2020) 11:6745-76. doi: 10.1039/D0FO01237H

43. Dajanta K, Apichartsrangkoon A, Chukeatirote E, Frazier RA. Free-amino acid profiles of thua nao, a Thai fermented soybean. Food Chem. (2011) 125:342-7. doi: 10.1016/j.foodchem.2010.09.002

44. Jin W, Zhang Z, Zhu K, Xue Y, Xie F, Mao S. Comprehensive understanding of the bacterial populations and metabolites profile of fermented feed by 16S rRNA gene sequencing and liquid chromatography-mass spectrometry. Metabolites. (2019) 9:239. doi: 10.3390/metabo9100239

45. Xu D, Wang P, Zhang X, Zhang J, Sun Y, Gao L, et al. Highthroughput sequencing approach to characterize dynamic changes of the fungal and bacterial communities during the production of sufu, a traditional Chinese fermented soybean food. Food Microbiol. (2020) 86:103340. doi: 10.1016/j.fm.2019.103340

46. Namgung HJ, Park HJ, Cho IH, Choi HK, Kwon DY, Shim SM, et al. Metabolite profiling of doenjang, fermented soybean paste, during fermentation. J Sci Food Agric. (2010) 90:1926-35. doi: 10.1002/jsfa. 4036

47. Shahzad R, Shehzad A, Bilal S, Lee IJ. Bacillus amyloliquefaciens RWL-1 as a new potential strain for augmenting biochemical and nutritional composition of fermented soybean. Molecules. (2020) 25:2346. doi: 10.3390/molecules 25102346

48. Roberts SA, Ball RO, Filler R, Moore AM, Pencharz PB. Phenylalanine and tyrosine metabolism in neonates receiving 
parenteral nutrition differing in pattern of amino acids. Pediatr Res. (1998) 44:907-14. doi: 10.1203/00006450-19981200000014

49. Kiriyama K, Hara KY, Kondo A. Oxidized glutathione fermentation using Saccharomyces cerevisiae engineered for glutathione metabolism. Appl Microbiol Biot. (2013) 97:7399-404. doi: 10.1007/s00253-0135074-8

50. Perin EC, Messias RD, Borowski JM, Crizel RL, Schott IB, Carvalho IR, et al. ABA-dependent salt and drought stress improve strawberry fruit quality. Food Chem. (2019) 271:516-26. doi: 10.1016/j.foodchem.2018. 07.213

51. Ai J, Wu QX, Battino M, Bai WB, Tian L. M. Using untargeted metabolomics to profile the changes in roselle (Hibiscus sabdariffa L) anthocyanins during wine fermentation. Food Chem. (2021) 364:130425. doi: 10.1016/j.foodchem.2021.130425

52. Coklo M, Maslov DR, Kraljević Pavelić S. Modulation of gut microbiota in healthy rats after exposure to nutritional supplements. Gut Microbes. (2020) 12:1-28. doi: 10.1080/19490976.2020.1779002

53. Owusu-Kwarteng J, Parkouda C, Adewumi GA, Ouoba LII, Jespersen L. Technologically relevant Bacillus species and microbial safety of west African traditional alkaline fermented seed condiments. Crit Rev Food Sci Nutr. (2020) 1-18. doi: 10.1080/10408398.2020.1830026

54. Ezeokoli O, Gupta A, Popoola T, Bezuidenhout C. Molecular analysis of bacterial community dynamics during the fermentation of soy-daddawa condiment. Food Sci Biotechnol. (2016) 25:10816. doi: 10.1007/s10068-016-0174-8

55. Hou, Q.C., Zhao, F.Y., Liu, W.J., Lv, R.R., Khine, W.W.T., Han, J., et al. (2020). Probiotic-directed modulation of gut microbiota is basal microbiome dependent. Gut Microbes. 2020, 12:1736974. doi: 10.1080/19490976.2020. 1736974

56. Hu YL, Dun YH, Li SN, Zhang DX, Peng N, Zhao SM, et al. Dietary Enterococcus faecalis LAB31 improves growth performance, reduces diarrhea, and increases fecal Lactobacillus number of weaned piglets. PLoS ONE. (2015) 10:e0116635. doi: 10.1371/journal.pone. 0116635
57. Foulquie Moreno MR, Sarantinopoulos P, Tsakalidou E, De Vuyst L. The role and application of enterococci in food and health. Int J Food Microbiol. (2006) 106:1-24. doi: 10.1016/j.ijfoodmicro.2005.06.026

58. Lopez-Gonzalez JA, Suarez-Estrella F, Vargas-Garcia MC, Lopez MJ, Jurado MM, Moreno J. Dynamics of bacterial microbiota during lignocellulosic waste composting: Studies upon its structure, functionality and biodiversity. Bioresour Technol. (2015) 175:406-16. doi: 10.1016/j.biortech.2014. 10.123

59. Xie WC, Song LY, Wang XY, Xu YG, Liu ZS, Zhao DF, et al. A bovine lactoferricin-lactoferrampin-encoding Lactobacillus reuteri CO21 regulates the intestinal mucosal immunity and enhances the protection of piglets against enterotoxigenic Escherichia coli K88 challenge. Gut Microbes. (2021) 13:1956281. doi: 10.1080/19490976.2021.1956281

60. Zong $\mathrm{X}, \mathrm{Fu} \mathrm{J}$, $\mathrm{Xu} \mathrm{BC}$, Wang $\mathrm{YZ}$, Jin ML. Interplay between gut microbiota and antimicrobial peptides. Anim Nutr. (2020) 6:389-96. doi: 10.1016/j.aninu.2020.09.002

Conflict of Interest: The authors declare that the research was conducted in the absence of any commercial or financial relationships that could be construed as a potential conflict of interest.

Publisher's Note: All claims expressed in this article are solely those of the authors and do not necessarily represent those of their affiliated organizations, or those of the publisher, the editors and the reviewers. Any product that may be evaluated in this article, or claim that may be made by its manufacturer, is not guaranteed or endorsed by the publisher.

Copyright (c) 2022 Wang, Wei, Jin, Liu, Yue and Wang. This is an open-access article distributed under the terms of the Creative Commons Attribution License (CC $B Y)$. The use, distribution or reproduction in other forums is permitted, provided the original author(s) and the copyright owner(s) are credited and that the original publication in this journal is cited, in accordance with accepted academic practice. No use, distribution or reproduction is permitted which does not comply with these terms. 